\title{
CNT Reinforced Hybrid Microgels as Scaffold Materials for Cell Encapsulation
}

Su Ryon Shin ${ }^{\dagger, \ddagger, 1}$, Hojae Bae ${ }^{\dagger, \ddagger, 1}$, Jae Min Cha ${ }^{\dagger, \neq, 1}$, Ji Young Mun§, Ying-Chieh Chen ${ }^{\perp}$, Halil Tekin $^{\dagger, \#, \|, \text { Hyeongho Shin }}{ }^{\dagger, \ddagger}$, Saeed Farshchi ${ }^{\Delta}$, Mehmet R. Dokmeci ${ }^{\dagger, \ddagger}$, Shirley Tang ${ }^{\Delta}$, and Ali Khademhosseini ${ }^{\dagger, \ddagger}$

†Center for Biomedical Engineering, Department of Medicine, Brigham and Women's Hospital, Harvard Medical School, 65 Landsdowne Street, Cambridge, MA 02139, USA.

¥Harvard-MIT Division of Health Sciences and Technology, Massachusetts Institute of Technology, Cambridge, MA 02139, USA.

$\S$ Department of Cell Biology, University of Massachusetts Medical School, 55 Lake Avenue North, Worcester, MA 01655, USA.

${ }^{\perp}$ Department of Applied Science, National Hsinchu University of Education, Hsinchu 300, Taiwan.

\#Department of Electrical Engineering and Computer Science, Massachusetts Institute of Technology, Cambridge, MA 02139, USA.

$\|$ David H. Koch Institute for Integrative Cancer Research, Massachusetts Institute of Technology, Cambridge, MA 02139, USA.

$\triangle$ Department of Chemistry, University of Waterloo, 200 University Ave. West, Waterloo, Ontario, N2L 3G1, Canada.

\section{Abstract}

Hydrogels that mimic biological extracellular matrix (ECM) can provide cells with mechanical support and signaling cues to regulate their behavior. However, despite the ability of hydrogels to generate artificial ECM that can modulate cellular behavior, they often lack the mechanical strength needed for many tissue constructs. Here, we present reinforced CNT-gelatin methacrylate (GelMA) hybrid as a biocompatible, cell-responsive hydrogel platform for creating cell-laden three dimensional (3D) constructs. The addition of CNTs successfully reinforced GelMA hydrogels without decreasing their porosity or inhibiting cell growth. The CNT-GelMA hybrids were also photopatternable allowing for easy fabrication of microscale structures without harsh processes. NIH-3T3 cells and human mesenchymal stem cells (hMSCs) readily spread and proliferated after encapsulation in CNT-GelMA hybrid microgels. By controlling the amount of CNTs incorporated into the GelMA hydrogel system, we demonstrated that the mechanical properties of the hybrid material can be tuned making it suitable for various tissue engineering applications. Furthermore, due to the high pattern fidelity and resolution of CNT incorporated GelMA, it can be used for in vitro cell studies or fabricating complex 3D biomimetic tissue-like structures.

Correspondence to: Ali Khademhosseini.

CORRESPONDING AUTHOR Center for Biomedical Engineering, Department of Medicine, Brigham and Women's Hospital, Harvard Medical School, 65 Landsdowne Street, Cambridge, MA 02139, USA, alik@ rics.bwh.harvard.edu (A. Khademhosseini). ${ }^{1}$ These authors contributed equally to this work.

Supporting Information Available: AFM images, Raman spectra, Optimization of UV exposure time for cell encapsulation, CNT toxicity test and cell adhesion on hydrogels. This material is available free of charge via the Internet at http://pubs.acs.org. 


\section{Keywords}

Carbon nanotubes; Cell-laden microgels; Tunable Stiffness; Biocompatibility; 3D tissue engineering

Extracellular matrix (ECM) is composed of networks of protein or polysaccharide-based nanofibers which provide mechanical support and signaling cues to cells. ${ }^{1,2}$ The interactions between the cells and the ECM have often been studied on two dimensional (2D) substrates. ${ }^{3-5}$ Despite the advantages of such 2D systems, which include well-controlled analysis of the cell-matrix interactions, they are limited in that the behavior of cells grown on a tissue culture substrate does not accurately represent that of the three dimensional (3D) tissue microenvironment. ${ }^{6-8}$ Therefore, the development of biomimetic $3 \mathrm{D}$ environments that allow for reconstruction of the complexity of the natural tissue is of great importance for enabling a range of applications in regenerative medicine and drug discovery. $3,9,10$

Hydrogels with their beneficial characteristics such as high water content and controllable biodegradability have been widely used as materials for generating 3D ECM scaffolds. In addition, the relative ease in modulating their physical and chemical properties and their high permeability for transport of nutrients and metabolites are also additional assets for cell encapsulation. ${ }^{11-15}$ Prior to using hydrogels in developing 3D ECM, it is desirable to control their mechanical properties so that they match the properties of the tissue they are aiming to emulate (brain, muscle, bone, etc). ${ }^{9}$ The mechanical properties of hydrogels can be controlled by a number of parameters such as the density and chemistry of the crosslinks and the concentration, chemistry and molecular weight of the precursors. However, it is observed that highly-crosslinked 3D hydrogel environments have high stiffnesses but limited cell proliferation, migration and morphogenesis. ${ }^{16-19}$ Therefore a method that enhances the mechanical properties of hydrogels without affecting their beneficial properties is needed for advanced engineered tissue constructs.

To enhance the mechanical properties of hydrogels, carbon nanotubes (CNTs) have been used as reinforcing agents to create porous hydrogels with tunable mechanical properties. Hybrid or composite materials based on CNTs have been shown to possess increased elastic modulus which combined the beneficial properties of multiple materials. ${ }^{20}$ For example, CNTs with polymer binders such as musselinspired polymer ${ }^{21}$ and DNA ${ }^{22}$ have been used to generate gels with exceptional mechanical properties through hydrophobic and $\pi-\pi$ interactions between the polymer binders and the CNT surface. Furthermore, conventional fabrication conditions used for generating CNT based gels require high $\mathrm{pH}$ and salt concentrations which are not compatible with cell encapsulation processes. ${ }^{23,24}$

To create a CNT reinforced hybrid hydrogel that can be used for 3D cell culture, we utilized photocrosslinkable gelatin methacrylate (GelMA) that has been shown to allow for cell encapsulation and proliferation. ${ }^{18}$ GelMA, which is a derivative of gelatin polypeptide, can also play the role of a biocompatible surfactant for the dispersion of nanotubes. GelMA contains RGD binding sequences and has bioactivity similar to that of collagen allowing cells to bind immediately to its surfaces. The protein structure of the GelMA hydrogels permits cells to enzymatically degrade and remodel the gel. ${ }^{19}$ In previous studies, GelMA was shown to create 3D cell-laden constructs with superior cell viability, spreading and migration. GelMA is also photopatternable allowing for easy fabrication of microscale structures. ${ }^{18}$ In addition, gelatin, which is similar to GelMA has been previously used in protecting aggregation of colloidal particles in suspension. ${ }^{25}$ The large surface area of the negatively and positively charged segments of GelMA interact with water and, together with the interactions between the hydrophobic segments of its polypeptide chain with nanotubes, 
can effectively coat and separate CNTs. ${ }^{26}$ Consequently, the GelMA coating, due to the hydrophobic interactions between the polypeptide chain and the CNT sidewalls is expected to enhance the mechanical stability of nanotubes. ${ }^{22}$

In this paper, we report a simple and direct method that uses GelMA-coated CNTs to reinforce hydrogels and demonstrate a biocompatible porous 3D scaffold with enhanced mechanical properties. We coated the CNTs with a thin layer of GelMA to improve their biological properties. We next incorporated the GelMA coated CNTs inside hydrogel structures and investigated the mechanical properties of these hybrid gels. We encapsulated cells inside these hybrid hydrogels and observed high levels of cellular viability, elongation and proliferation. The CNT-GelMA hybrid hydrogel system with tunable mechanical properties and enhanced cellular behavior may be useful as a scaffolding material for 3D tissue engineering constructs.

\section{Results and Discussion}

The first goal of this study was to generate bio-functionalized CNTs by coating CNT with GelMA. In general, materials for cell-laden hydrogels have been prepared in DPBS or media which are similar to the biological environment. Cell culture media contains various salts (concentration: $\sim 0.15 \mathrm{M}$ ) within which CNTs precipitate. This observation is characteristic of a lyophobic colloid which is stabilized through electrostatic repulsion. ${ }^{27}$ In our work, by coating the CNTs with a thin layer of GelMA, we were able to successfully disperse them in DPBS solution (Figure 1 (a)). The HRTEM data shows the morphological differences between bare and GelMA-coated CNTs, indicating that a thin layer of GelMA was uniformly coated on the CNT surface (Figure 1 (b) and (c)). To confirm the dispersion of the CNTs in medium, we first removed the CNTs from the medium and after being dried, analyzed the CNTs by AFM to measure their diameter and length distributions. In terms of the diameters, bare CNTs were measured to be $\sim 9 \pm 8 \mathrm{~nm}$ whereas GelMA-coated CNTs were found to be $\sim 25 \pm 12 \mathrm{~nm}$ (Figure 1 (d)). The measured diameter increase with AFM agree with HRTEM results. On the other hand, the lengths of CNTs were not significantly affected by the GelMA-coating, since similar length distributions were observed with and without GelMA coating (see Supporting Information, Figure S1). Therefore, GelMA coating did not cause significant crosslinking of CNTs and rendered well dispersed CNTs in PBS.

In general, CNT functionalization processes via chemical or physical treatments induce defects on CNTs and decrease their electrical and mechanical properties. Using Raman spectroscopy, we confirmed that our process did not cause significant cutting or structural damage to CNTs. The ratio between the two characteristic peaks of CNT, which are the Dband around $1300 \mathrm{~cm}^{-1}$ and the G- band at $1592 \mathrm{~cm}^{-1}$, is commonly used as an indicator of CNT defect density. This ratio is about the same in the Raman spectra of bare CNTs and GelMA coated CNTs (see Supporting Information, Figure S2). The relatively short sonication time $(\sim 1 \mathrm{hr})$ and high viscosity of GelMA solution might have been beneficial in preserving the structural integrity of CNTs. The strong broadband background observed in the spectrum of GelMA coated CNTs can be attributed to the luminescence of GelMA. ${ }^{28}$

To characterize the structure of GelMA on the surface of CNTs, CD was conducted to analyze the polypeptide backbone conformations. The strong negative peak at $198 \mathrm{~nm}$ shown in Figure 1(e) indicates that GelMA adopted a typical gelatin conformation similar to a random coil structure. ${ }^{29}$ As gelatin is derived by breaking the triple-helix structure of collagen, the amplitude of the positive peak at $220 \mathrm{~nm}$, characteristic of the triple-helix, had almost disappeared. However, GelMA showed a decrease in the amplitude of the negative peak at $198 \mathrm{~nm}$ when compared to gelatin, which may be caused by the methacrylated pendant groups on its polypeptide backbone (degree of methacrylation: 75\%). Moreover, the 
spectrum of the signal obtained from the GelMA coated CNTs had lower peak amplitude observed at $198 \mathrm{~nm}$. In a previous study, a random coil peptide upon interacting with nanoparticles had a change in the secondary structure which was observed by the decrease in the negative peak at $198 \mathrm{~nm}^{29}$, which is similar to our observations. It was reported that proteins have the ability to strongly bind onto CNT surface due to the hydrophobic interaction. ${ }^{30}$ It may be that the polypeptide chains of the GelMA were disturbed during the sonication process for the preparation of GelMA-coated CNTs, and subsequently reoriented on the CNT surface through hydrophobic interactions ( $\sim 50 \%$ hydrophobic residue in gelatin chains). ${ }^{26,31}$ The large surface area of the hydrophilic segments of GelMA interact with water and together with the interactions between the hydrophobic segments of its polypeptide chain with nanotubes can effectively coat and separate CNTs. The thin GelMA layer on coated CNTs not only increased the solubility of CNTs in DPBS and other biological media but also provided large numbers of acrylic groups on CNT surfaces. Both factors are important to achieve enhancement of mechanical properties CNT-GelMA hybrid hydrogels.

The GelMA-coated CNTs were well dispersed in the prepolymer solution without any evidence of aggregation as shown in Figure 2 (a) with a more uniform dispersion compared to a previous study of single-walled carbon nanotube (SWNT) loaded collagen I-Matrigel ${ }^{\mathrm{TM}}$ composite scaffolds (concentration of CNT: $50 \mu \mathrm{g} / \mathrm{ml}$ ). ${ }^{32}$ In Figure 2 (b), HRTEM analysis shows the well dispersed CNTs in the prepolymer solution. The absorbance of the CNTloaded prepolymer solution is important in this study. Higher CNT concentrations resulted in higher UV absorbance during GelMA-CNT propolymer solution UV crosslinking step and therefore longer UV exposure time was necessary for adequate crosslinking. We also investigated the absorbance of the CNT-based prepolymer solution versus the concentration of CNTs ( 0 to $1 \mathrm{mg} / \mathrm{ml})$. The darkness of the prepolymer solution increased in proportion to an increase in the concentration of CNTs (Figure 2 (a)) and the absorbance of the prepolymer solution increased almost linearly corresponding to an increase in the concentration of nanotubes measured at $280 \mathrm{~nm}$ (the wavelength of excitation for the photoinitiator) (Figure 2(c)). It is worthwhile to mention that the nanotubes in the prepolymer solution tend to absorb UV light at this wavelength. Therefore, the excitation of the photoinitiator can be interrupted by the CNTs or the darker color, hence the CNT-based prepolymer solution may require a much longer polymerization time to form a gel than the prepolymer solution without CNTs. We have previously demonstrated micropatterning of GelMA hydrogels with a controlled size and without using cytotoxic reagents such as sodium dodecyl sulfate (SDS). ${ }^{33}$ In this work, we extended on our previous work for patterning of CNT-GelMA microstructures. By labeling the CNTs with a fluorescent dye (FITC), we were able to create well defined microscale gels with a uniform dispersion of CNTs inside. The fluorescence signals obtained from the gels indicated a homogeneous distribution of CNTs without phase separation (Figure 2 (d)).

To successfully employ the CNT-GelMA hybrid as a suitable 3D material for tissue engineering applications, cells were encapsulated inside the hybrid gels and their behavior were subsequently investigated. NIH-3T3 fibroblasts were encapsulated in CNT-GelMA hybrid micropatterns with varying concentrations of CNTs from 0 to $0.5 \mathrm{mg} / \mathrm{ml}$. To encapsulate cells inside microscale hydrogels that have similar number of cells in all samples, the UV exposure time to crosslink was optimized based on the number of encapsulated cells and their viability (see Supporting Information, Figure S3). As reported before, cells encapsulated in hydrogels tend to have a uniform distribution with high viability within the $3 \mathrm{D}$ structure. ${ }^{19}$ General loss of cellular viability may occur due to stress during the encapsulation procedure or oxygen and nutrient limitations due to molecular diffusion. However, after optimization of the encapsulation and cell culture processes, we successfully decreased the populations of dead cells and obtained greater than $90 \%$ cellular 
viability for $48 \mathrm{hr}$ in all groups (Figure 3 (a) - (f) and (g)). Interestingly, the cell viability was inversely proportional to longer exposure times in CNT-GelMA hydrogel with increased the concentration of CNT. In photoencapsulation process, the two main factors affecting cell viability are UV exposure conditions and photoinitiator concentration that produce free radicals when exposed to the light of appropriate wavelength. ${ }^{34}$ It has been reported that UV light alone at low intensity $\left(6 \mathrm{~mW} / \mathrm{cm}^{2}\right.$ or $\left.4 \mathrm{~mW} / \mathrm{cm}^{2}\right)$ for several minutes did not affect cell viability significantly. ${ }^{35}$ Therefore, the exposure times used to adequately crosslink CNT-GelMA $(30-80 \mathrm{sec})$ will not have significant effect on cell viability. Moreover, CNTs have been reported to be effective scavengers of radical oxygenated species through electron-transfer processes and adduct formation. ${ }^{36}$ Specifically, surplus free radicals after reaction of carbon-carbon double bonds from the methacrylate groups in CNT-GelMA solution can attach to the nanotube surfaces. Consequently, the encapsulated cells in CNT-GelMA hydrogels showed high viability even after longer UV exposure times. To verify the potential cytotoxicity of CNTs, a cellular viability test was performed within a medium in which either bare or GelMA-coated CNTs were suspended (see Supporting Information, Figure S4). In these results, no significant cytotoxicity was observed in the suspension of GelMA-coated CNTs up to a CNT concentration of $50 \mu \mathrm{g} / \mathrm{ml}$. Moreover, the viable cell population in the suspension of $50 \mu \mathrm{g} / \mathrm{ml}$ bare CNTs compared to GelMA coated CNT suspension was found to be significantly lower similar to a trend reported previously. ${ }^{37}$ In a previous study, the use of chemically modified CNTs which were treated with acidic reagents were found to decrease cellular viability. ${ }^{37} \mathrm{We}$ also evaluated cellular viability after seeding NIH-3T3 fibroblasts onto a CNT-GelMA substrate with varying concentrations of CNTs and demonstrated that cells maintained their viability during $48 \mathrm{hr}$ of culture while spreading well over the substrates (see the Supporting Information, Figure S5).

To evaluate cellular proliferation inside the hydrogels, an MTS assay was employed to measure the metabolic activity during the culture period (Figure $3(\mathrm{~h})$ ). Cells in all groups including a bare GelMA hydrogel showed no significant difference in the proliferation rates for the first $24 \mathrm{hr}$. From $24 \mathrm{hr}$ to $48 \mathrm{hr}$, the cell numbers more than doubled. Interestingly, the cells encapsulated in the CNT-containing hydrogels proliferated faster than those in the GelMA-only hydrogel controls. Thus, the GelMA-coated CNTs did not display visible cytotoxic effects to cells under various culture conditions including GelMA-coated CNTs suspended in aqueous media, on 2D CNT-GelMA substrates, and encapsulated inside 3D CNT-GelMA hydrogels. Furthermore, the GelMA-coated CNT incorporation into the GelMA hydrogels appeared to support cellular growth with a higher cellular proliferation rate compared to GelMA only hydrogels. ${ }^{32}$ In previous studies, ECM functionalized CNT showed stronger cell adhesion compared to ECM only and resulted in higher cellular proliferation rate and supported guided cell growth through strong affinity between CNT and ECM proteins. ${ }^{38-40}$ The GelMA exhibited strong affinity to CNTs and still maintained functional structure even after coating onto the CNTs. Therefore, the higher cellular proliferation rate is possible arising from occur due to stronger cell adhesion than GelMA only.

To determine the effect of incorporating CNTs on the mechanical properties of GelMA hydrogels, unconfined compression and tensile tests were performed with samples swollen in DPBS. The CNT-GelMA hydrogels demonstrated significantly stronger mechanical properties than GelMA-only hydrogels particularly in terms of tensile strength (Figure 4 (a) and (b) and Table S1). The elastic modulus of the hydrogels increased after the incorporation of CNTs from $15 \mathrm{kPa}$ (bare GelMA) to $\sim 60 \mathrm{kPa}$ when $0.5 \mathrm{mg} / \mathrm{ml}$ of CNTs were incorporated. The CNT-GelMA hybrid hydrogels displayed high toughness and strong tensile strength and still maintained their ability to elongate compared to bare GelMA hydrogels. The increase in compression strength by the addition of CNTs was only marginal as shown in the results from a hydrogel containing $0.5 \mathrm{mg} / \mathrm{ml}$ of CNTs (116 $\mathrm{kPa})$ compared 
to a bare GelMA hydrogel (100 kPa) (Figure 4 (c)). A significant difference in the compressive modulus was found only at $0.5 \mathrm{mg} / \mathrm{ml}$ of CNTs $(\sim 30 \mathrm{kPa})$, while the other concentrations of CNTs and the bare GelMA hydrogels had values between 10 and $18 \mathrm{kPa}$. Interestingly, the CNT-GelMA hydrogels appeared to show a higher compressive modulus than CNT-DNA sponge fibers $(\sim 10 \mathrm{kPa})$ that were formed without nanofiber junctions but included CNTs concentration that were ten-times higher. ${ }^{20}$ The compressive modulus of the hybrid hydrogels containing $0.5 \mathrm{mg} / \mathrm{ml}$ of CNTs showed mechanical properties similar to those found in native tissue environments such as muscle $(8-17 \mathrm{kPa})$ and osteoid matrix $(25-40 \mathrm{kPa})$. The GelMA only gels showed a higher elongation to break (72\%) than that of the CNT-incorporated ones (58\%) suggesting that the rigid reinforcements created by the CNTs may have limited the compressive deformation of the elastic elements in the hydrogel. ${ }^{41}$ It was reported in a previous study that the strong nanofiber junctions in the electrospun fabrics resulted in mechanically malleable as well as durable characteristics of the structure showing a low elastic modulus and high toughness. ${ }^{42}$ Paper is another well known microporous material where the mechanical properties are determined by the strength and density of nanofiber junctions. ${ }^{20}$ The strong adhesion between GelMA coated on CNTs to the acrylic group on GelMA chains resulted in the reinforcement of mechanical properties of the hybrid CNT-GelMA hydrogels. This assumption is supported by our observations in the SEM images of CNT-GelMA hydrogel cross sections. The highly microporous structure of GelMA hydrogels, as shown in the SEM micrographs (Figure 5), resemble the parallel sheets composed of a collection of polyhedral cells as gelatin forms a sheet-like structure while frozen. ${ }^{43}$ Interestingly, however, the size of the pores appeared to increase with increasing CNT concentrations. The porosity of GelMA hydrogels did not appear to be affected by the addition of CNTs (Figure 5 (c)) except for $0.5 \mathrm{mg} / \mathrm{ml} \mathrm{CNT-GelMA} \mathrm{hybrid}$ hydrogels. Furthermore, the overall performance of the CNT-GelMA was not affected. The surface of pore walls, which are the concentrated polymeric domain, appeared smooth without aggregated CNTs, indicating that almost all the CNTs were embedded inside the polymer matrix. We also observed groups of entangled nanofibers in the pores with diameters in the range of 50 to $100 \mathrm{~nm}$ as seen in Figure 5 (d) and (e). The chemical crosslinking of acrylic groups on GelMA-coated CNT surfaces result in nanofiber junctions. Therefore, the crosslinked GelMA-coated CNT chemical network offered an improvement in the mechanical stability of the hydrogel. However, the nanofiber junctions based on CNTs mostly influenced the tensile strength of the hybrid hydrogels since the nanotubes, due to their high aspect ratio, could easily buckle upon experiencing the compressive load whereas they were more durable against tensile force where the fibers align in parallel with the pulling force. ${ }^{41}$

The mechanical properties of the scaffold material including the porosity of the matrix environment are recognized to affect cellular behavior such as proliferation, function, and differentiation. For example, an interesting study performed on hydrogel substrates with different stiffnesses, soft (elastic modulus: $\sim 1 \mathrm{kPa})$ and stiff $(30 \sim 100 \mathrm{kPa})$, revealed that the cellular morphology was affected by the mechanical properties of the substrates that the cells were cultured on. ${ }^{44}$ Therefore, it is of great interest to tune the mechanical properties of hydrogels while preserving their beneficial characteristics for tissue engineering applications. In general, the mechanical properties of hydrogels can be engineered by varying hydrogel concentrations. We have previously reported that increasing the concentration of GelMA up to $15 \%$ could lead to reinforcement in the mechanical properties of hydrogels $(30 \mathrm{kPa})$; however, the increased polymer concentration caused a denser pore structure in the matrix which resulted in limited degradability and permeability characteristics for the hydrogels and impeded cellular migration and morphogenesis. ${ }^{3,} 18$ In Figure 6 (a) and (b), the encapsulated 3T3 fibroblast cells displayed their normal growth and spreading patterns in CNT-GelMA hydrogel similar to results obtained in softer GelMA hydrogels. However, the encapsulated 3T3 fibroblast cells did not display their normal 
growth and spreading patterns in 10\% (Figure $6(\mathrm{c})$ ) and 15\% (data not shown) GelMA hydrogels as expected from the previous study. ${ }^{18}$ It was elucidated experimentally that the $10 \%$ GelMA hydrogel had a denser pore structure compared with 5\% GelMA and CNTGelMA hydrogels (see the Supporting Information, Figure S6). Similarly, in several other studies higher polymer concentrations were used to create mechanically reinforced hydrogels, and cells encapsulated in these hydrogels such as PEG-GelMA ( $80 \mathrm{kPa})$ and PEG-HA ( 45 kPa) demonstrated slow cellular growth and limited spreading ability due to the high concentration of the polymers (Table 1). ${ }^{16,19,45,46}$ Therefore, it is worthwhile to report that the addition of CNTs in GelMA hydrogels, while successfully reinforcing their mechanical characteristics (up to $30 \mathrm{kPa}$ ) compared to bare GelMA hydrogels $(\sim 10 \mathrm{kPa})$, preserved their porous structure. We examined the spreading patterns of the cells inside hybrid hydrogels after $48 \mathrm{hr}$ of culture by staining the actin filament networks with Alexa488-conjugated phalloidin (Figure 6 (d) and (e)). 3T3 cells inside CNT-GelMA hydrogels displayed elongated, spread and well-interconnected cellular shapes similar to results obtained from softer GelMA hydrogels.

To demonstrate the feasibility of using CNT-GelMA hybrid hydrogels for other tissue engineering applications, hMSCs were encapsulated in these hydrogels. Chang et al. has demonstrated that hMSC showed different morphology depending on ECM stiffness. ${ }^{4} \mathrm{We}$ studied the morphology of encapsulated hMSCs in $0.5 \mathrm{mg} / \mathrm{ml} \mathrm{CNT-GelMA} \mathrm{hybrid}$ hydrogels using an SEM. The SEM observations performed at high magnifications revealed the morphology of physical contacts between the cells and the CNT based nanofiber junctions, as shown in Figure 7 (a) and (b). Round cell bodies measured to be approximately 7- $10 \mu \mathrm{m}$ in diameter were observed in the gels. Spherical hMSCs in CNT-GelMA hydrogels were hung by long threadlike cytoplasmic prolongations, similar to observations reported for hMSCs cultured on CNT substrates (Figure 7 (b)). ${ }^{47}$ A larger number of cytoplasmic prolongations were found in CNT-GelMA hydrogels compared to GelMA-only gels. Alternatively, the flat cell body of hMSCs in pure GelMA grew shorter cytoplasmic prolongations that adhered to the more evenly distributed layer of GelMA (Figure 7 (a)). Nanometer scale cytoplasmic prolongations were not observed in hMSCs grown inside pure GelMA hydrogels (Figure 7 (a)). In addition, the enzyme-mediated degradation profiles demonstrated that the degradation rates can be tuned by varying the amount of incorporated CNTs in hydrogels (see Supporting Information, Figure S7). The incorporated nanotubes can act as water insoluble backbones hindering the permeation of collagenase into the hydrogels. The cells spread and expanded in the degraded spaces of the hydrogel which was observed by a fluorescence cell tracker and GelMA functionalized with FITC-tagged CNTs.

\section{Conclusions}

In this study, CNTs were successfully coated with a thin layer of GelMA by using the hydrophobic interactions between the polypeptide chains of the GelMA and the sidewalls of the nanotubes while still possessing their intact physical characteristics. The GelMA coating enabled homogeneous dispersion of CNTs in a GelMA prepolymer solution. We also demonstrated photopatterning of CNT-GelMA hybrid hydrogel with controllable dimensions and shapes. The cytotoxicity tests indicated that the majority of the encapsulated cells in the CNT-GelMA hydrogels were highly viable. The mechanical properties of CNTGelMA hybrid hydrogels were enhanced by the addition of CNTs. The nanofiber junctions formed inside the hybrid hydrogels were created by the covalent bonding between acrylic groups on GelMA-coated CNT surfaces. The nanofiber web-like structures formed by the CNTs in the hydrogels served as an enhancer for mechanical properties while maintaining the beneficial bioactive properties of the GelMA material as a scaffold which are important attributes for in vitro tissue development such as the inclusion of bioactivity, high porosity, and degradability. The CNT-GelMA hybrids showed promising biocompatibility results 
with hMSCs and appeared to modulate the cell morphology. In addition, with their tunable mechanical stiffness with porous structure, they are expected to find niche applications in creating artificial 3D ECM scaffolds to guide hMSC differentiation to osteoblastic, neural lineages and skeletal muscular in a manner dependent on their stiffness.

\section{Experimental Methods}

\section{Materials}

Carboxyl functionalized multi-walled carbon nanotubes (diameter $\sim 9 \pm 8 \mathrm{~nm}$ and 50-250 $\mu \mathrm{m}$ in length; 95\% purity) from NanoLab Inc. were used as purchased. Gelatin (Type A, 300 bloom from porcine skin), polyethylene glycol diacrylate (PEGDA), 3-(trimethoxysily) propyl methacrylate (TMSPMA), and methacrylic anhydride (MA) were purchased from Sigma-Aldrich (Wisconsin, USA). Photomasks were purchased from CAD Art (Washington, USA). The UV curing system (Omnicure S2000) was fabricated by EXPO Photonic Solutions Inc. (Ontario, Canada).

\section{Gelatin methacrylate synthesis}

GelMA was synthesized as described previously. ${ }^{18}$ Briefly, gelatin was added at $10 \%(\mathrm{w} / \mathrm{v})$ into Dulbecco's phosphate buffered saline (DPBS) at $50{ }^{\circ} \mathrm{C}$ and stirred until dissolved. MA $\left(0.8 \mathrm{ml}\right.$ per $g$ gelatin) was then added to the gelatin solution while stirring at $50{ }^{\circ} \mathrm{C}$ for $2 \mathrm{hr}$. The ratio of reacted lysine groups was modified by changing the amount of MA in the initial reaction mixture. To stop the reaction, the mixture was diluted with DPBS. The diluted mixture was subsequently dialyzed against distilled water for 1 week at $40^{\circ} \mathrm{C}$ to remove unreacted MA and salt (Molecular weight cut off: $12-14 \mathrm{kDa}$ ). The solution was lyophilized for 1 week.

\section{Preparation of CNT-GeIMA hybrid hydrogels}

Lyophilized GelMA (2\%) was dissolved for $30 \mathrm{~min}$ in DPBS at $80^{\circ} \mathrm{C}$. CNTs $(5 \mathrm{mg} / \mathrm{ml})$ were then added to this solution and sonicated (VCX 400, $80 \mathrm{~W}, 2 \mathrm{sec}$ on and $1 \mathrm{sec}$ off) for $1 \mathrm{hr}$ in a water bath to obtain a black ink-like solution. To prepare the prepolymer solution, the GelMA-coated CNTs with the target concentration of CNTs $(0,0.1,0.25$ and $0.5 \mathrm{mg} / \mathrm{ml})$ were added to the GelMA solution (5\%) containing $0.5 \%$ photoinitiator, 2-hydroxy-1-(4(hydroxyethoxy) phenyl)-2-methyl-1-propanone (Irgacure 2959; CIBA chemical). The absorbance of the CNT-GelMA prepolymer solution with various concentrations of CNTs were obtained with a UV spectrophotometer (Shimadzu, UV-2450, Japan). To fabricate micropatterned CNT-GelMA hydrogel, photomasks with arrays of $300 \mu \mathrm{m}$ square pattern and other patterns were designed using AutoCAD. To produce microscale CNT-GelMA hydrogels, the UV exposure time was optimized for each concentration of CNTs as shown in Figure S3 (see Supporting Information).

\section{Characterization of the GelMA-coated CNTs}

High resolution transmission electron microscope (HRTEM) images (Tecnai 12, FEI, Netherlands) were acquired using a charge coupled detector (CCD) camera. All sample solutions were loaded onto holey-carbon film-supported grids with negative staining (Uranyl acetate). Raman analysis were carried out using a Jobin Yvon LabRam Model HR800 Raman microscope equipped with an Ar-ion excitation laser $(\lambda=514.523 \mathrm{~nm}$, power $=0.5$ $\mathrm{mW})$. A $50 \times$ microscope objective was used to focus the laser beam and to collect the scattered light. CNTs, as purchased and GelMA-coated, were deposited on $\mathrm{SiO}_{2}$ substrates pretreated with 3-amino-propyl trimethoxysilane (APTMS) and imaged using an atomic force microscope (AFM) (Multimode IV, Veeco, USA). Coating of APTMS on $\mathrm{SiO}_{2}$ surfaces was performed by immersion of the dry substrates into a dilute solution of APTMS 
in methanol (5\% in volume) for $24 \mathrm{hr}$ at room temperature. To remove physisorbed APTMS, the substrates were rinsed thoroughly with methanol and treated under sonication for $5 \mathrm{~min}$ in methanol. The substrates were rinsed with ultrapure water and then blown dry with pure $\mathrm{N}_{2}$. The substrates were then placed in an oven at $120^{\circ} \mathrm{C}$ for $30 \mathrm{~min}$. All circular dichroism (CD) spectra were recorded on a spectropolarimeter (Aviv Instruments, model 202,

Proterion Corporation, Pistacaway, NJ) equipped with a programmable temperature control unit. CD spectra were collected with a scan speed of $12 \mathrm{~nm} / \mathrm{min}$ and each spectrum was then averaged for two scans. The CD spectra were carried out at a sample concentration of 0.1 $\mathrm{mg} / \mathrm{ml}$ at a path length of $1 \mathrm{~mm}$. The wavelength-dependence changes in the PMT voltage, which was often ignored in previous $\mathrm{CD}$ studies of nanoparticle/protein interactions, rose rapidly below $195 \mathrm{~nm}$ and caused noise in the CD spectra, compared to longer wavelengths. ${ }^{30}$ The recorded spectra in millidegrees of ellipticity $(\ominus)$ were converted to mean residue ellipticity $[\Theta]$ in deg. $\mathrm{cm}^{2} \cdot \mathrm{dmol}^{-1}$ by the following equation: $[\Theta]=(\ominus \times 100 \times$ $\left.\mathrm{M}_{\mathrm{r}}\right) /\left(\mathrm{c} \times I \times \mathrm{N}_{\mathrm{A}}\right)$, where $\mathrm{c}$ is the protein concentration in $\mathrm{mg} / \mathrm{ml}, l$ is the path length in $\mathrm{cm}$, $\mathrm{M}_{\mathrm{r}}$ is the protein molecular weight and $\mathrm{N}_{\mathrm{A}}$ is the number of proteins.

\section{Mechanical testing}

To analyze the mechanical properties of the gels, $50 \mu \mathrm{l}$ of a prepolymer solution was placed between two glass slides separated by a $650 \mu \mathrm{m}$ spacer and exposed to UV light $(6.9 \mathrm{~mW} /$ $\mathrm{cm}^{2}, 360-480 \mathrm{~nm}$ ) for specific times. Samples were incubated in DPBS at room temperature for $24 \mathrm{hr}$ and then detached from the slide. The compression tests were performed with a swollen hydrogel disc sample, $6 \mathrm{~mm}$ in diameter and $700 \mu \mathrm{m}$ in thickness. The hydrogel disc was tested at a rate of $20 \%$ strain/min on an Instron 5524 mechanical analyzer (Instron, Canton, MA) for compression testing. The compressive modulus was calculated from the slope in the linear region corresponding to $5-15 \%$ strain. The tensile modulus was tested with a rectangular shape hydrogel $(1 \times \mathrm{w} \times \mathrm{t}=7 \mathrm{~mm} \times 2.3 \mathrm{~mm} \times 0.6 \mathrm{~mm})$ at $50 \%$ swollen state and at a rate of $10 \%$ strain/min. The samples were stretched to their breaking point. The tensile modulus was calculated from the slope in the linear region corresponding to 0 $10 \%$ strain. $^{48}$ The sample size was 5 gels per group.

\section{Scanning electron microscope (SEM) Imaging}

The structure of the CNT-GelMA hydrogel was imaged using an SEM (Hitachi Model S4700, Japan). The swollen hydrogels were frozen with liquid nitrogen and then lyophilized. The lyophilized samples were cut to expose their cross-sections and were coated with Pt/Pd using a sputter coater. The quantification of the porosity based on the SEM pictures was analyzed by NIH ImageJ software. Six images for each group were used for the quantification. For analysis of SEM image of encapsulated cells in the hydrogel, human mesenchymal stem cells (hMSCs) were grown for 14 days and fixed in $4 \%$ paraformaldehyde for $20 \mathrm{~min}$ at room temperature. The samples were washed with DPBS and frozen at liquid nitrogen and then lyophilized.

\section{Cell encapsulation}

NIH-3T3 fibroblasts were cultured in high glucose Dulbecco's Modified Eagle Medium (DMEM) supplemented with 10\% FBS and $1 \%$ penicillin-streptomycin in a $5 \% \mathrm{CO}_{2}$ atmosphere at $37^{\circ} \mathrm{C}$ and passaged 2 times per week. NIH-3T3 fibroblasts were trypsinized and resuspended in CNT-GelMA prepolymer solution containing $0.5 \%$ photoinitiator at a concentration of $5 \times 10^{6}$ cells $/ \mathrm{ml}$. The glass slides were coated with PEGDA (20\%) on TMSPMA coated glass slide to inhibit cell adhesion. Microgel units $(300 \mu \mathrm{m}(\mathrm{w}) \times 300 \mu \mathrm{m}(I) \times$ $150 \mu \mathrm{m}(\mathrm{h})$ ) were fabricated on PEGDA coated glass slides as previously described following exposure to $6.9 \mathrm{~mW} / \mathrm{cm}^{2} \mathrm{UV}$ light for 30, 40, 60 and 80s with CNT concentrations of $0,0.1,0.25$ and $0.5 \mathrm{mg} / \mathrm{ml}$, respectively. ${ }^{18}$ The glass slides containing microgels were washed with cell culture medium and incubated in DMEM containing FBS 
and penicillin-streptomycin standard culture conditions. The hMSCs were cultured in $\operatorname{MSCGM}^{\mathrm{TM}}$ (Lonza) for 5 days in a $5 \% \mathrm{CO}_{2}$ atmosphere at $37^{\circ} \mathrm{C}$. The culture medium was changed every 3 days during the experiments. The hMSCs were trypsinized and resuspended in 5\% GelMA (control) and $0.5 \mathrm{mg} / \mathrm{ml} \mathrm{CNT-GelMA} \mathrm{prepolymer} \mathrm{solution} \mathrm{containing} 0.5 \%$ photoinitiator at a concentration of $5 \times 10^{6}$ cells $/ \mathrm{ml}$. Cell-laden hydrogels $(6 \mathrm{~mm}$ in diameter and $0.7 \mathrm{~mm}$ in thickness) were fabricated by exposure to $6.9 \mathrm{~mW} / \mathrm{cm}^{2} \mathrm{UV}$ light for 30 and 80s with $5 \%$ GelMA and $0.5 \mathrm{mg} / \mathrm{ml}$ CNT-GelMA, respectively.

\section{Cell characterization}

A calcein-AM/ethidium homodimer Live/Dead assay (Invitrogen) was used according to the manufacturer's instructions. The Live/Dead image was measured using an inverted fluorescence microscope (Nikon, Eclipse TE 2000U, Japan). The number of live and dead cells from 10 randomly selected microgel units of three patterns of each group was measured by ImageJ software. To determine the proliferation of cells inside CNT-GelMA hydrogels, MTS assays were performed according to the manufacturer's instructions. For immunostaining, encapsulated cells were washed with DPBS and fixed in $4 \%$ paraformaldehyde for $20 \mathrm{~min}$ at room temperature. After fixation, cells were washed with DPBS and then treated with $0.15 \%$ Triton X-100 in DPBS for 10 min. Cells were stained with Alexa Fluor 594 phalloidin at 1:100 dilution in blocking buffer for $40 \mathrm{~min}$ and counterstained with DAPI at 1:20000 dilution in DPBS for $40 \mathrm{~min}$. The samples were measured by inverted laser scanning confocal Microscope (Leica SP5X MP, Germany).

\section{Hydrogel degradation}

The hydrogels were placed in $1.5 \mathrm{ml}$ tubes with $1 \mathrm{ml}$ of DPBS with $1 \mathrm{U} / \mathrm{ml}$ of collagenase type II (Worthington Biochemical). Gels were incubated with enzyme at $37^{\circ} \mathrm{C}$ for $1.5,3,6$, $9,12,18$, or $24 \mathrm{hr}$. After incubation for the specific duration, the remaining hydrogel was washed with DPBS, all of the liquid was removed and the gels were lyophilized. The percent degradation was calculated by dividing the weight of the dried hydrogels to the weight of the untreated hydrogels. To visualize the degradation of CNT-GelMA hydrogels, we tracked the movement of CNTs inside the hydrogels. The CNTs (carboxyl functionalized) were covalently bonded to ethylene diamine and then the amine functionalized CNTs were treated with FITC (green color). The encapsulated cells were pre-labeled with a Cell tracker CMFDA (Invitrogen) according to manufacturer's instructions.

\section{Statistical analysis}

Statistical significance was measured by performing a one-way or two-way ANOVA where appropriate (GraphPad Prism 5.02, GraphPad Software). Tukey's multiple comparison test was used to determine whether a significant difference exists between specific treatments. Differences were taken to be significant for $\mathrm{p}<0.05$.

\section{Supplementary Material}

Refer to Web version on PubMed Central for supplementary material.

\section{Acknowledgments}

SR Shin was partially supported by the National Research Foundation of Korea Grant funded by the Korean Government (Ministry of Education, Science and Technology) [NRF-2010-357- D00069]. This paper was supported by the Institute for Soldier Nanotechnology, National Institutes of Health (HL092836, EB02597, AR057837, HL099073), the National Science Foundation (DMR0847287) and the Office of Naval Research Young Investigator award. 


\section{REFERENCES}

1. Engler AJ, Sen S, Sweeney HL, Discher DE. Matrix Elasticity Directs Stem Cell Lineage Specification. Cell. 2006; 126:677-689. [PubMed: 16923388]

2. Discher DE, Janmey P, Wang Y-1. Tissue Cells Feel and Respond to the Stiffness of their Substrate. Science. 2005; 310:1139-1143. [PubMed: 16293750]

3. Lutolf MP, Gilbert PM, Blau HM. Designing Materials to Direct Stem-Cell Fate. Nature. 2009; 462:433-441. [PubMed: 19940913]

4. Park JS, Chu JS, Tsou AD, Diop R, Tang Z, Wang A, Li S. The Effect of Matrix Stiffness on the Differentiation of Mesenchymal Stem Cells in Response to TGF- $\beta$. Biomaterials. 2011; 32:39213930. [PubMed: 21397942]

5. Byfield FJ, Wen Q, Levental I, Nordstrom K, Arratia PE, Miller RT, Janmey PA. Absence of Filamin a Prevents Cells from Responding to Stiffness Gradients on Gels Coated with Collagen but not Fibronectin. Biophys J. 2009; 96:5095-5102. [PubMed: 19527669]

6. Tibbitt MW, Anseth KS. Hydrogels as Extracellular Matrix Mimics for 3D Cell Culture. Biotechnology and Bioengineering. 2009; 103:655-663. [PubMed: 19472329]

7. Petersen OW, Ronnovjessen L, Howlett AR, Bissell MJ. Interaction with Basement-Membrane Serves to Rapidly Distinguish Growth and Differentiation Pattern of Normal and Malignant Human Breast Epithelial Cells. Proceedings of the National Academy of Sciences. 1992; 89:9064-9068.

8. Tanaka H, Murphy CL, Kimura M, Kawai S, Polak JM. Chondrogenic Differentiation of Murine Embryonic Stem Cells: Effects of Culture Conditions and Dexamethasone. Journal of Cellular Biochemistry. 2004; 93:454-462. [PubMed: 15372628]

9. Kumachev A, Greener J, Tumarkin E, Eiser E, Zandstra PW, Kumacheva E. High-Throughput Generation of Hydrogel Microbeads with Varying Elasticity for Cell Encapsulation. Biomaterials. 2011; 32:1477-1483. [PubMed: 21095000]

10. Derda R, Tang SK, Laromaine A, Mosadegh B, Hong E, Mwangi M, Mammoto A, Ingber DE, Whitesides GM. Multizone Paper Platform for 3D Cell Cultures. PLoS One. 2011; 6:e18940. [PubMed: 21573103]

11. Chia SM, Leong KW, Li J, Xu X, Zeng KY, Er PN, Gao SJ, Yu H. Hepatocyte Encapsulation for Enhanced Cellular Functions. Tissue Engineering. 2000; 6:481-494. [PubMed: 11074935]

12. Bienaime C, Barbotin JN, Nava-Saucedo JE. How to Build an Adapted and Bioactive Cell Microenvironment? A Chemical Interaction Study of the Structure of Ca-Alginate Matrices and their Repercussion on Confined Cells. Journal of Biomedical Materials Research Part A. 2003; 67A:376-388. [PubMed: 14566778]

13. Uludag H, De Vos P, Tresco PA. Technology of Mammalian Cell Encapsulation. Advanced Drug Delivery Reviews. 2000; 42:29-63. [PubMed: 10942814]

14. Drury JL, Mooney DJ. Hydrogels for Tissue Engineering: Scaffold Design Variables and Applications. Biomaterials. 2003; 24:4337-4351. [PubMed: 12922147]

15. Peppas NA, Hilt JZ, Khademhosseini A, Langer R. Hydrogels in Biology and Medicine: from Molecular Principles to Bionanotechnology. Advanced Materials. 2006; 18:1345-1350.

16. Patel PN, Gobin AS, West JL, Patrick CW Jr. Poly(ethylene glycol) Hydrogel System Supports Preadipocyte Viability, Adhesion, and Proliferation. Tissue Eng. 2005; 11:1498-1505. [PubMed: 16259604]

17. Engler AJ, Sen S, Sweeney HL, Discher DE. Matrix Elasticity Directs Stem Cell Lineage Specification. Cell. 2006; 126:677-689. [PubMed: 16923388]

18. Nichol JW, Koshy ST, Bae H, Hwang CM, Yamanlar S, Khademhosseini A. Cell-laden Microengineered Gelatin Methacrylate Hydrogels. Biomaterials. 2010; 31:5536-5544. [PubMed: 20417964]

19. Hutson CB, Nichol JW, Aubin H, Bae H, Yamanlar S, Al-Haque S, Koshy ST, Khademhosseini A. Synthesis and Characterization of Tunable Poly(Ethylene Glycol): Gelatin Methacrylate Composite Hydrogels. Tissue Eng Part A. 2011; 17:1713-1723. [PubMed: 21306293]

20. Lee CK, Shin SR, Mun JY, Han SS, So I, Jeon JH, Kang TM, Kim SI, Whitten PG, Wallace GG, et al. Tough Supersoft Sponge Fibers with Tunable Stiffness from a DNA Self-Assembly Technique. Angew Chem Int Ed Engl. 2009; 48:5116-5120. [PubMed: 19263454] 
21. Ryo S, Lee Y, Hwang J-W, Hong S, Kim C, Park TG, Lee H, Hong SH. High-Strength Carbon Nanotube Fibers Fabricated by Infiltration and Curing of Mussel-Inspired Catecholamine Polymer. Advanced Materials. 2011; 23:1971-1975. [PubMed: 21523834]

22. Shin SR, Lee CK, So I, Jeon J-H, Kang TM, Lee C, Kim SI, Spinks GM, Wallace GG, Kim SJ. DNA-Wrapped Single-walled Carbon Nanotube Hybrid Fibers for Supercapacitors and Artificial Muscles. Advanced Materials. 2008; 20:466-470.

23. Yadav SK, Bera T, Saxena PS, Maurya AK, Garbyal RS, Vajtai R, Ramachandrarao P, Srivastava A. MWCNTs as Reinforcing Agent to the Hap $>$ Gel Nanocomposite for Artificial Bone Grafting. Journal of Biomedical Materials Research Part A. 2010; 93A:886-896. [PubMed: 19705464]

24. Kwon J, Kim H. Comparison of the Properties of Waterborne Polyurethane/Multiwalled Carbon Nanotube and Acid-treated Multiwalled Carbon Nanotube Composites Prepared by in situ Polymerization. Journal of Polymer Science Part A: Polymer Chemistry. 2005; 43:3973-3985.

25. Nabeta M, Sano M. Nanotube Foam Prepared by Gelatin Gel as a Template. Langmuir. 2005; 21:1706-1708. [PubMed: 15723462]

26. Liu Y, Liu X, Wang X. Biomimetic Synthesis of Gelatin Polypeptide-Assisted Noble-Metal Nanoparticles and their Interaction Study. Nanoscale Research Letters. 2011; 5:22-32.

27. Li D, Muller MB, Gilje S, Kaner RB, Wallace GG. Processable Aqueous Dispersions of Graphene Nanosheets. Nature Nanotechnology. 2008; 3:101-105.

28. Huang W, Taylor S, Fu K, Zhang D, Hanks TW, Rao AM, Sun Y-P. Attaching Proteins to Carbon Nanotubes via Diimide-Activated Amidation. Nano Letters. 2002; 2:311-314.

29. Ali MS, Anjum K, Khan JM, Khan RH, Kabir ud D. Complexation Behavior of Gelatin with Amphiphilic Drug Imipramine Hydrochloride as Studied by Conductimetry, Surface Tensiometry and Circular Dichroism Studies. Colloids Surf B Biointerfaces. 2011; 82:258-262. [PubMed: 20870394]

30. Mu Q, Liu W, Xing Y, Zhou H, Li Z, Zhang Y, Ji L, Wang F, Si Z, Zhang B, et al. Protein Binding by Functionalized Multiwalled Carbon Nanotubes is Governed by the Surface Chemistry of both Parties and the Nanotube Diameter. The Journal of Physical Chemistry C. 2008; 112:3300-3307.

31. Karajanagi SS, Yang H, Asuri P, Sellitto E, Dordick JS, Kane RS. Protein-Assisted Solubilization of Single-walled Carbon Nanotubes. Langmuir. 2006; 22:1392-1395. [PubMed: 16460050]

32. Behan BL, DeWitt DG, Bogdanowicz DR, Koppes AN, Bale SS, Thompson DM. Single-Walled Carbon Nanotubes alter Schwann Cell Behavior Differentially within 2D and 3D Environments. J Biomed Mater Res A. 2011; 96:46-57. [PubMed: 20949573]

33. Aubin H, Nichol JW, Hutson CB, Bae H, Sieminski AL, Cropek DM, Akhyari P, Khademhosseini A. Directed 3D Cell Alignment and Elongation in Microengineered Hydrogels. Biomaterials. 2010; 31:6941-6950. [PubMed: 20638973]

34. Williams CG, Malik AN, Kim TK, Manson PN, Elisseeff JH. Variable Cytocompatibility of Six Cell Lines with Photoinitiators used for Polymerizing Hydrogels and Cell Encapsulation. Biomaterials. 2005; 26:1211-1218. [PubMed: 15475050]

35. Fedorovich NE, Oudshoorn MH, van Geemen D, Hennink WE, Alblas J, Dhert WJ. The Effect of Photopolymerization on Stem Cells Embedded in Hydrogels. Biomaterials. 2009; 30:344-353. [PubMed: 18930540]

36. Galano A. Carbon Nanotubes as Free-Radical Scavengers. Journal of Physical Chemistry C. 2008; 112:8922-8927.

37. Magrez A, Kasas S, Salicio V, Pasquier N, Seo JW, Celio M, Catsicas S, Schwaller B, Forro L. Cellular Toxicity of Carbon-Based Nanomaterials. Nano Lett. 2006; 6:1121-1125. [PubMed: 16771565]

38. Abdul Kafi M, El-Said WA, Kim TH, Choi JW. Cell Adhesion, Spreading, and Proliferation on Surface Functionalized with RGD Nanopillar Arrays. Biomaterials. 2011:1-9. Online Published.

39. Baik KY, Park SY, Heo K, Lee KB, Hong S. Carbon Nanotube Monolayer Cues for Osteogenesis of Mesenchymal Stem Cells. Small. 2011; 7:741-745. [PubMed: 21425459]

40. Namgung S, Kim T, Baik KY, Lee M, Nam JM, Hong S. Fibronectin-Carbon-Nanotube Hybrid Nanostructures for Controlled Cell Growth. Small. 2011; 7:56-61. [PubMed: 21061404] 
41. Spinks GM, Shin SR, Wallace GG, Whitten PG, Kim SI, Kim SJ. Mechanical Properties of Chitosan/CNT Microfibers Obtained with Improved Dispersion. Sensors and Actuators B. 2006; 115:678-685.

42. Kidoaki S, Kwon IK, Matsuda T. Mesoscopic Spatial Designs of Nano- and Microfiber Meshes for Tissue-Engineering Matrix and Scaffold Based on Newly Devised Multilayering and Mixing Electrospinning Techniques. Biomaterials. 2005; 26:37-46. [PubMed: 15193879]

43. Vlierberghe SV, Cnudde V, Dubruel P, Masschaele B, Cosijns A, Paepe ID, Jacobs PJ, Hoorebeke LV, Remon JP, Schacht E. Porous Gelatin Hydrogels: 1. Cryogenic Formation and Structure Analysis. Biomacromolecules. 2007; 8:331-337. [PubMed: 17291055]

44. Reilly GC, Engler AJ. Intrinsic Extracellular Matrix Properties Regulate Stem Cell Differentiation. J Biomech. 2010; 43:55-62. [PubMed: 19800626]

45. Hwang NS, Varghese S, Li H, Elisseeff J. Regulation of Osteogenic and Chondrogenic Differentiation of Mesenchymal Stem Cells in PEG-ECM Hydrogels. Cell Tissue Res. 2011; 344:499-509. [PubMed: 21503601]

46. Hwang CM, Sant S, Masaeli M, Kachouie NN, Zamanian B, Lee SH, Khademhosseini A. Fabrication of Three-Dimensional Porous Cell-Laden Hydrogel for Tissue Engineering. Biofabrication. 2010; 2035003.

47. Zanello LP, Zhao B, Hu H, Haddon RC. Bone Cell Proliferation on Carbon Nanotubes. Nano Lett. 2006; 6:562-567. [PubMed: 16522063]

48. Kim SJ, Spinks GM, Prosser S, Whitten PG, Wallace GG, Kim SI. Surprising Shrinkage of Expanding Gels under an External Load. Nat Mater. 2006; 5:48-51. [PubMed: 16380727] 
(a)

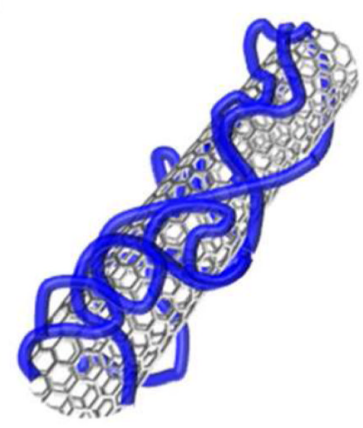

(b)

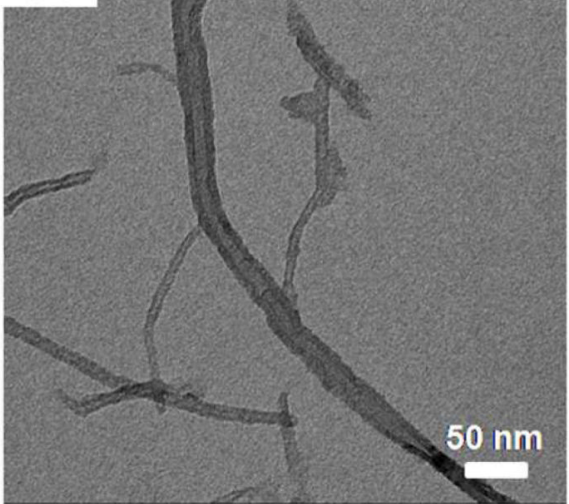

(c)

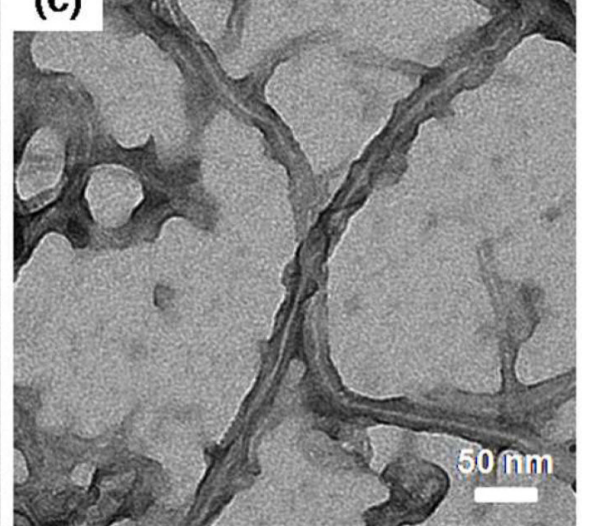

(d)

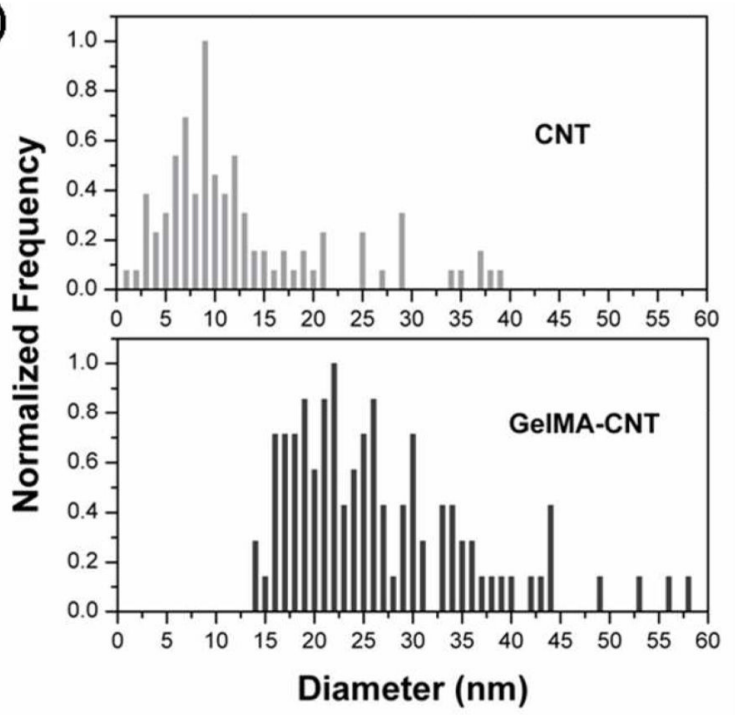

(e)

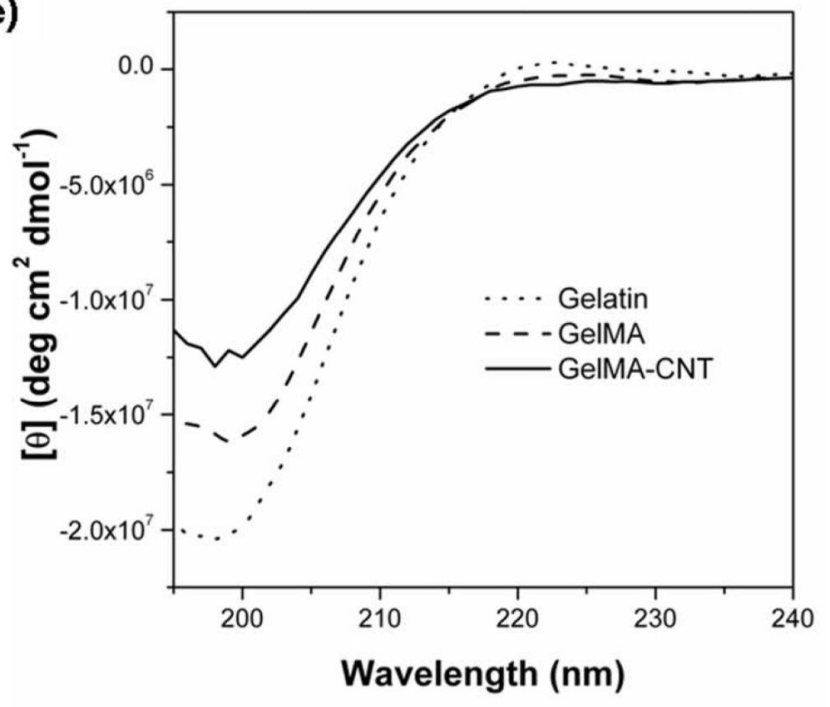

Figure 1.

(a) Schematic diagram of a GelMA-coated CNT. The drawing is only a graphical representation and does not represent the precise means through which GelMA polypeptide chains (blue lines) interact with the CNT surface. HRTEM images of (b) bare CNTs and (c) GelMA-coated CNTs. (d) Diameter distributions of bare CNTs and GelMA-coated CNTs. (e) Change in CD spectra of gelatin, GelMA and GelMA-coated CNTs. 


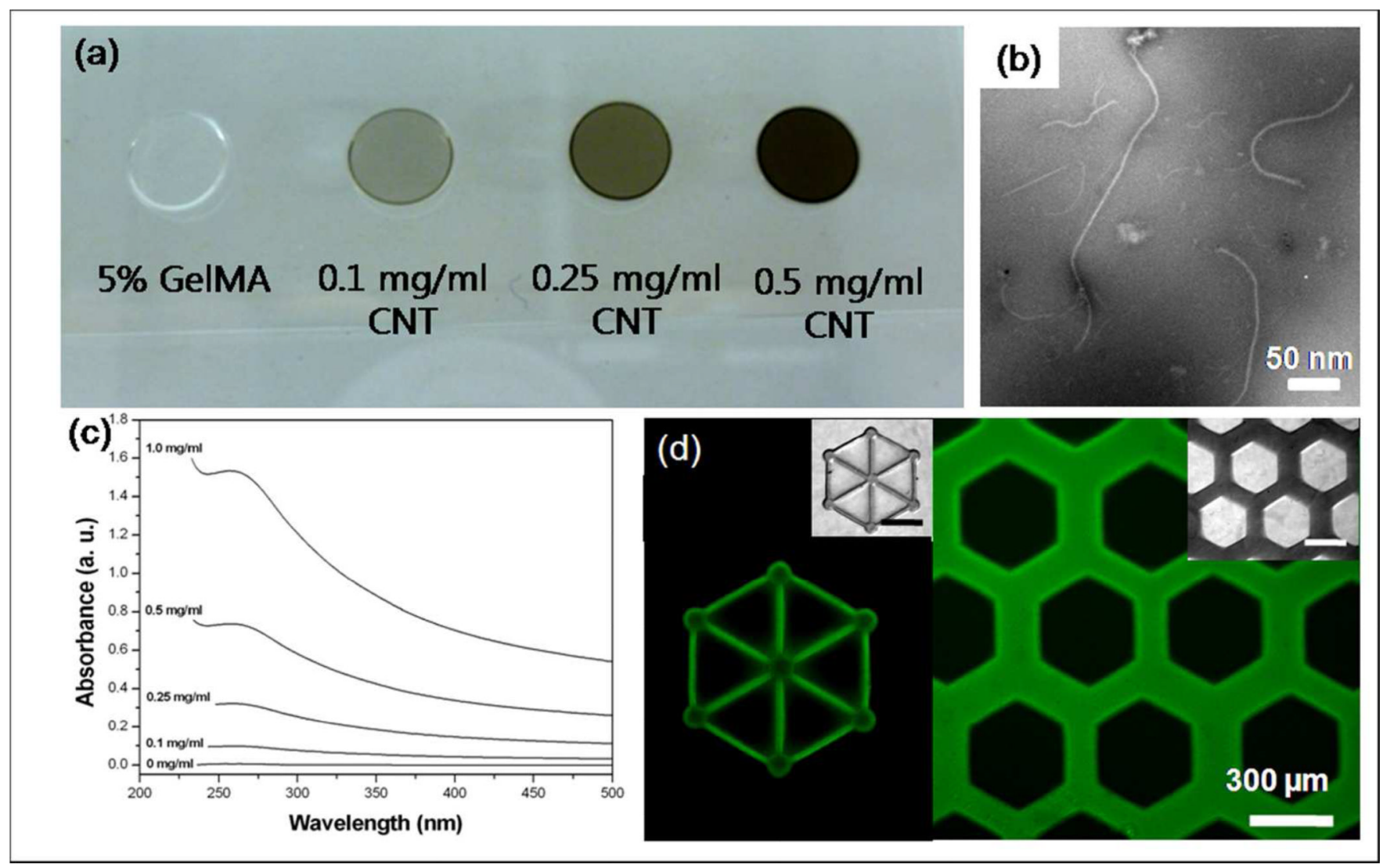

Figure 2.

(a) Optical images of CNT-GelMA prepolymer solutions with different concentrations of CNTs. (b) HRTEM images of well dispersed CNT-GelMA prepolymer solutions with 0.5 $\mathrm{mg} / \mathrm{ml} \mathrm{CNTs}$. (c) UV-Vis absorption spectra of CNT-GelMA prepolymer solutions with different concentrations of CNTs. (d) Fluorescence images of micropatterned CNT-GelMA hydrogels with GelMA coated FITC-functionalized CNTs. Inset Figures are phase contrast images (scale bar $=300 \mu \mathrm{m}$ ). 

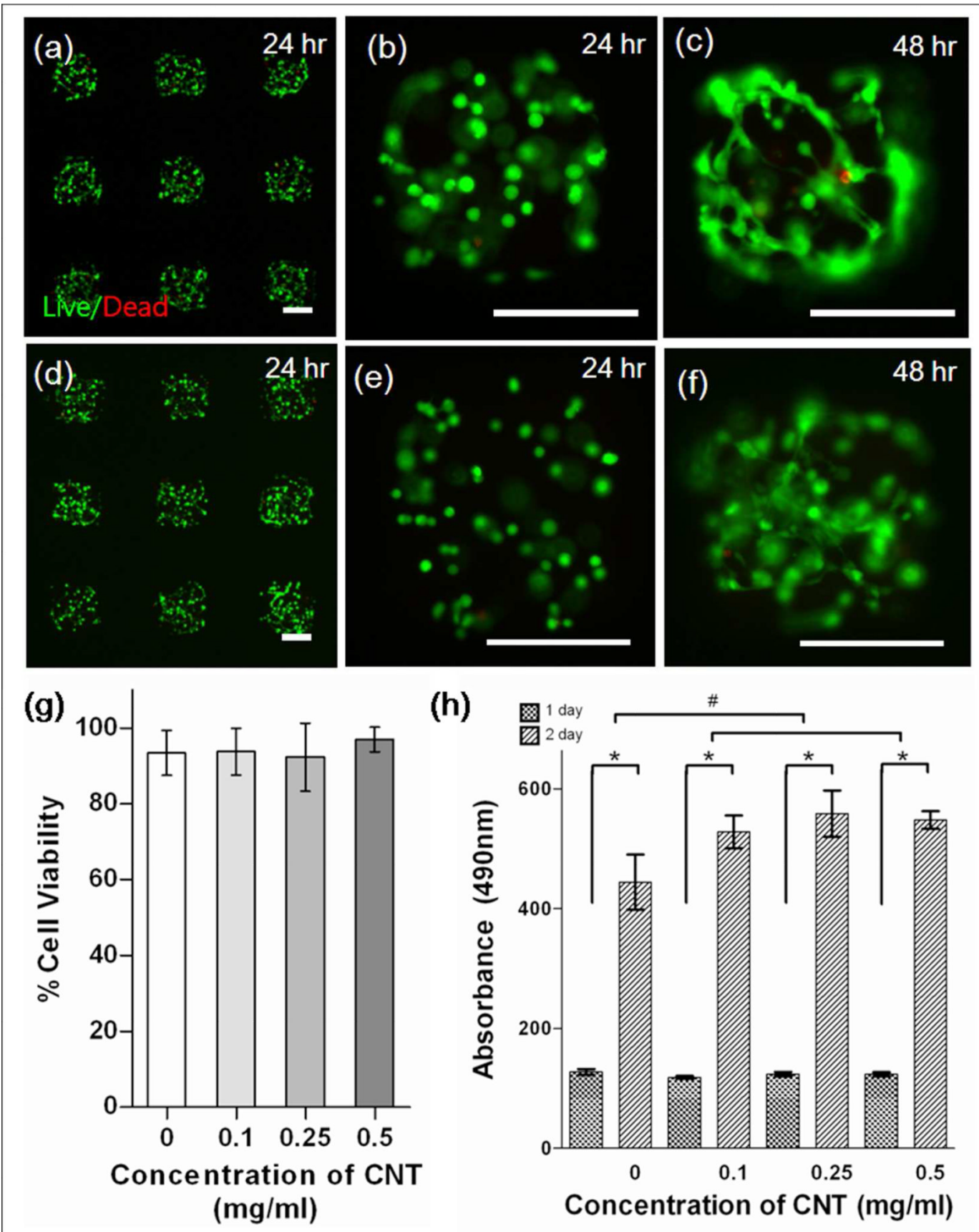

(h)

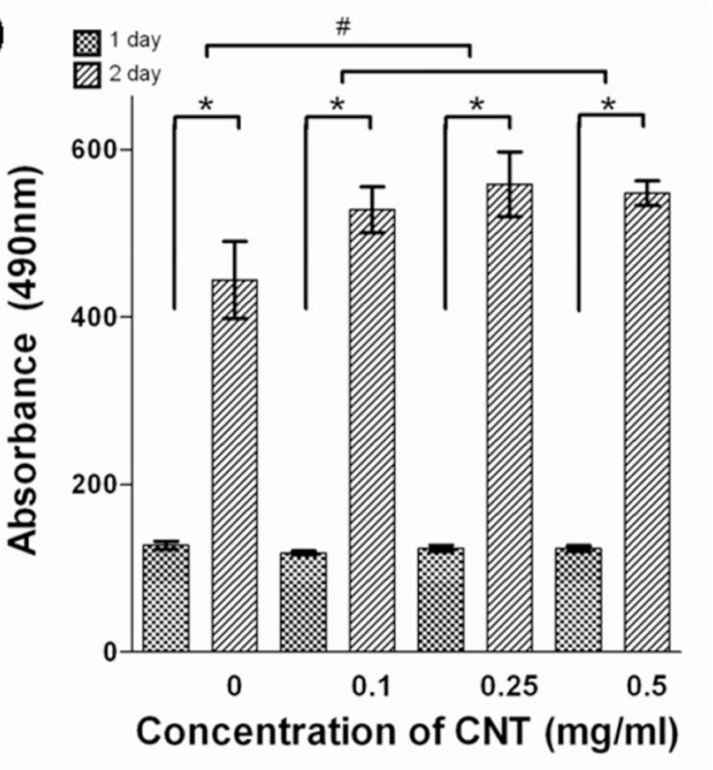

Figure 3.

Representative images of 3T3 fibroblasts embedded in GelMA ( $(\mathrm{a}-\mathrm{c}))$ and CNT-GelMA hydrogel $(0.5 \mathrm{mg} / \mathrm{ml})$ micropatterns $((\mathrm{d}-\mathrm{f}))$ which were stained with calcein-AM (green) / ethidium homodimer (red) Live/Dead assay $24 \mathrm{hr}$ and $48 \mathrm{hr}$ after encapsulation. (scale bar = $250 \mu \mathrm{m})(\mathrm{g})$ Quantification of cell viability demonstrated excellent cell survival for all conditions after $24 \mathrm{hr}$ of encapsulation. Error bars represent the standard deviation (SD) of averages obtained from 10 images with 3 independent samples from each condition. (h) MTS assay of the cells encapsulated in micorpatterned GelMA and CNT-GelMA hydrogels after $24 \mathrm{hr}$ and $48 \mathrm{hr}$. Statistical analyses were performed by two-way ANOVA. Asterisks 
$(*)$ and hash (\#) marks indicate the significant difference $(\mathrm{P}<0.05)$ in the time periods and experimental groups respectively. 

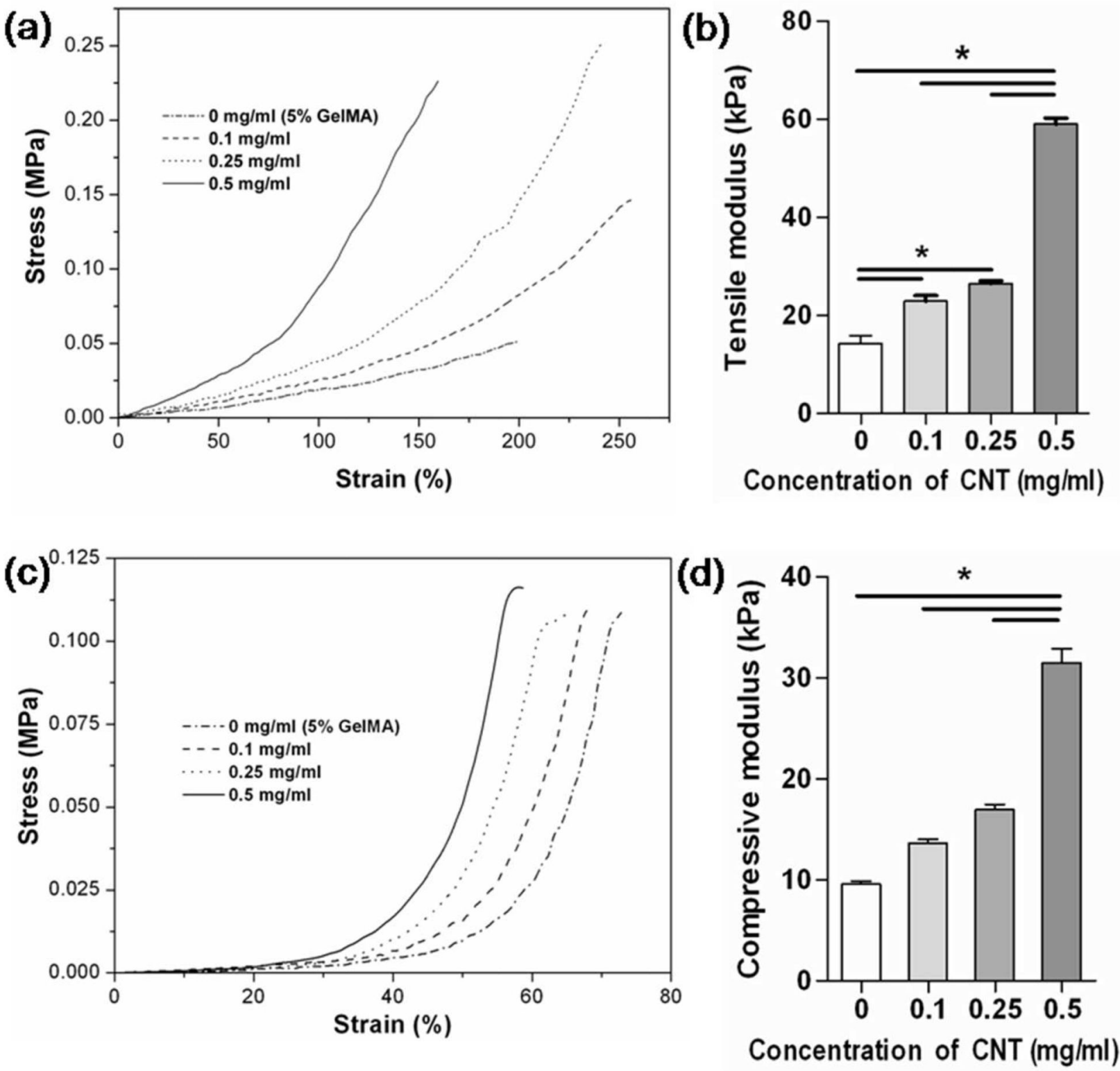

Figure 4.

The mechanical properties of CNT-GelMA hydrogels with varying concentrations of CNTs. (a) Representative curves of tension tests and (b) elastic modulus at 50\% swelling ratio. (c) Stress and strain curves of compression tests and (d) elastic modulus at fully swollen state. Error bar represents the SD of measurements performed on 5 samples. The elastic modulus was significantly different $(* p<0.05)$ between GelMA and CNT-GelMA hydrogels. 


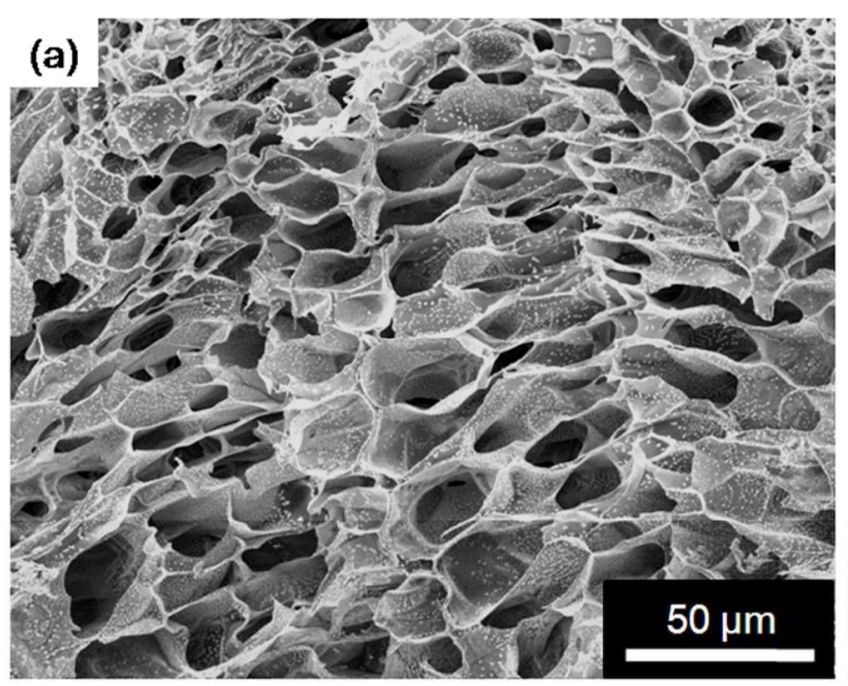

(c)

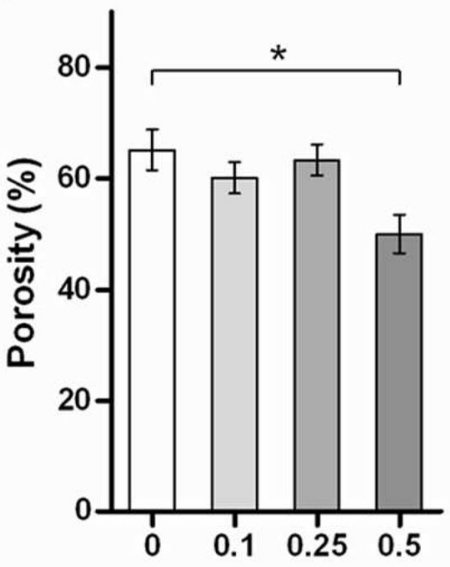

Concentration of CNT $(\mathrm{mg} / \mathrm{ml})$

Figure 5. on 6 samples.

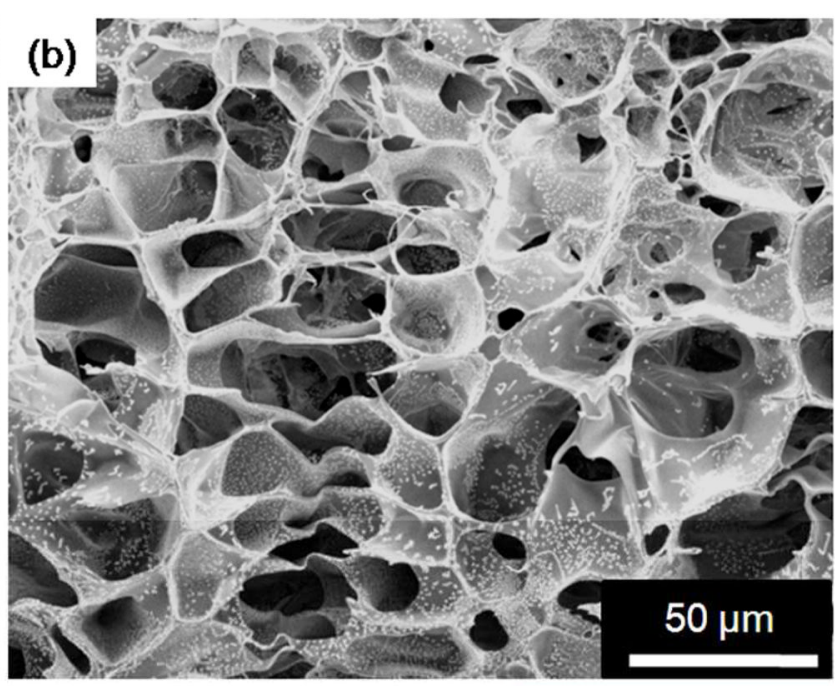

(d)

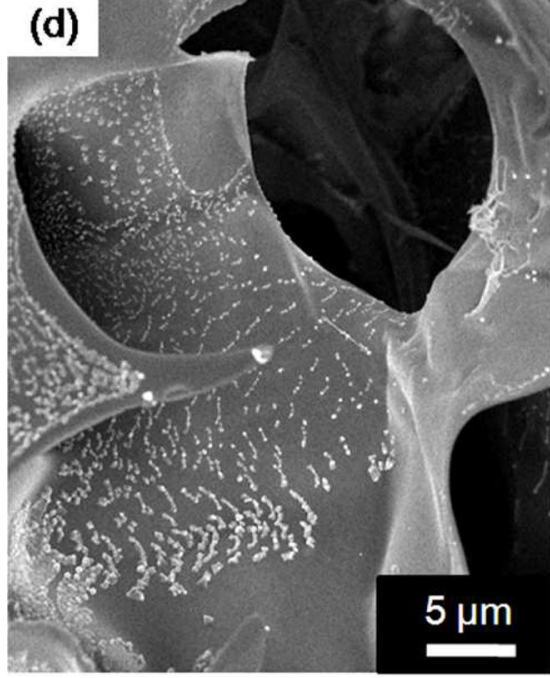

(e)

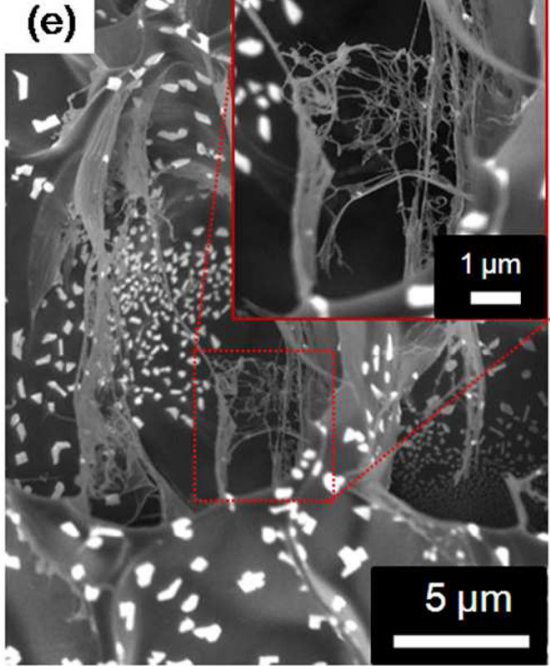

SEM images of cross sections of (a) GelMA and (b) CNT-GelMA hydrogels with $0.5 \mathrm{mg} / \mathrm{ml}$ CNTs. (c) The porosity of the CNT-GelMA hydrogels with different concentrations of CNTs quantified by ImageJ based on the SEM images $(* p<0.05)$. Magnified images show parts of a cross section of (d) GelMA and (e) CNT-GelMA hydrogel with nanofiber junctions inside a porous structure. Error bars represent the SD of measurements performed 


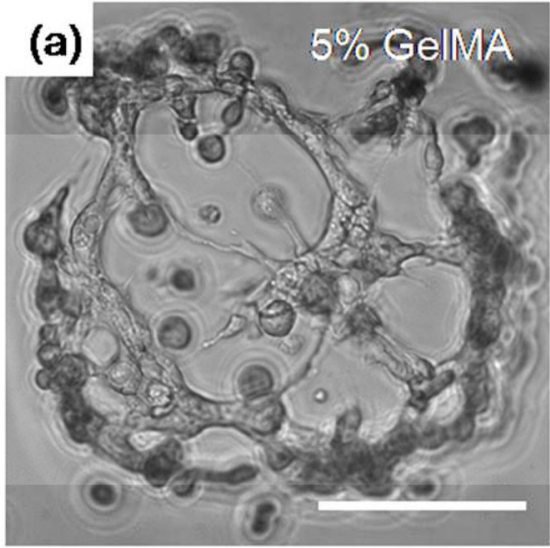

(d)
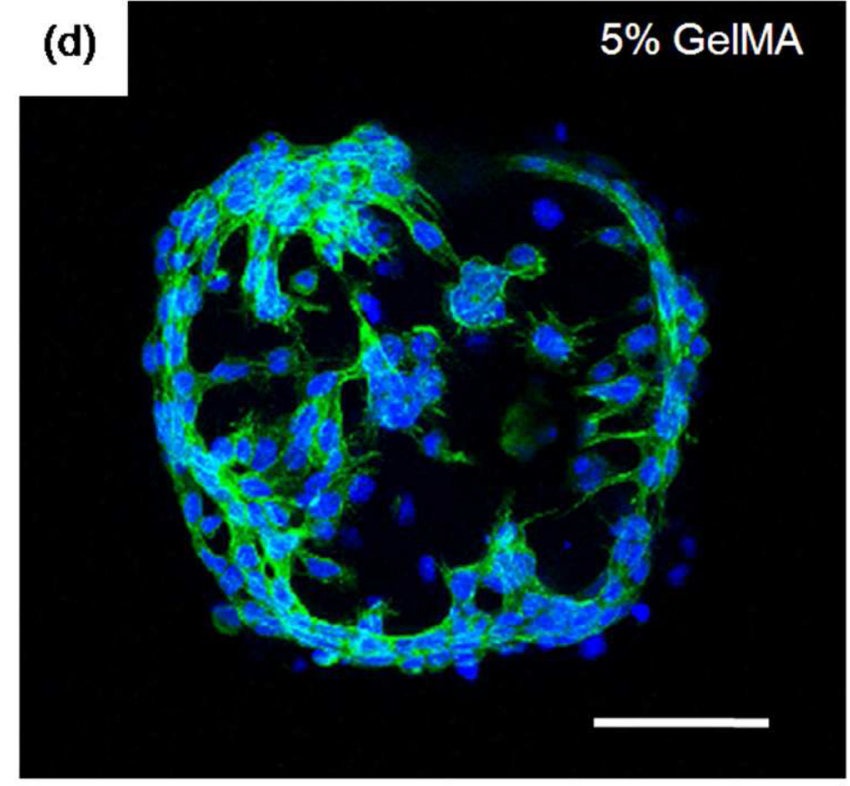

(b) $0.5 \mathrm{mg} / \mathrm{m} / \mathrm{CNT}$ - GellMA
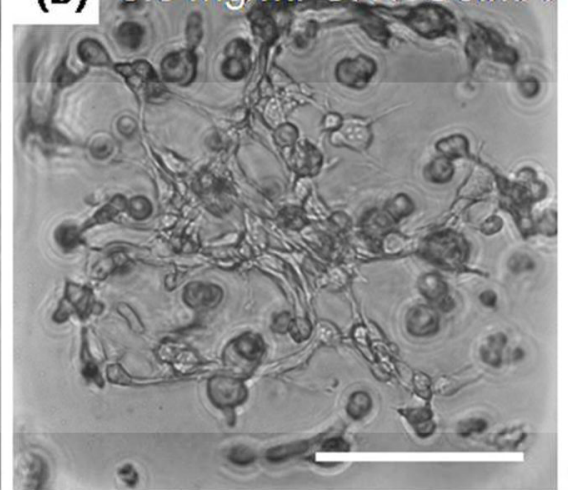

$5 \%$ GelMA

\section{(e)}

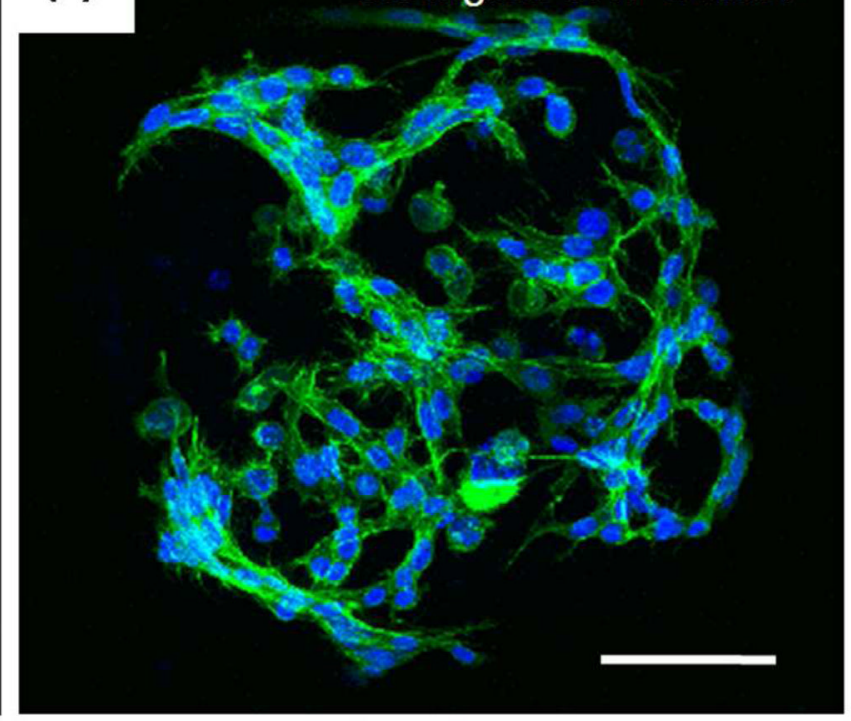

Figure 6.

Cell spreading in (a) 5\% GelMA, (b) CNT-GelMA hydrogel $(0.5 \mathrm{mg} / \mathrm{ml})$, and (c) $10 \%$ GelMA after $48 \mathrm{hr}$ in culture. Fluorescence images of cells forming interconnected networks in micropatterened (d) 5\% GelMA and (e) CNT-GelMA hybrid hydrogels after $48 \mathrm{hr}$ with Phalloidin /DAPI staining of F-actin/cell nuclei. (scale bar $=100 \mu \mathrm{m}$ ) 


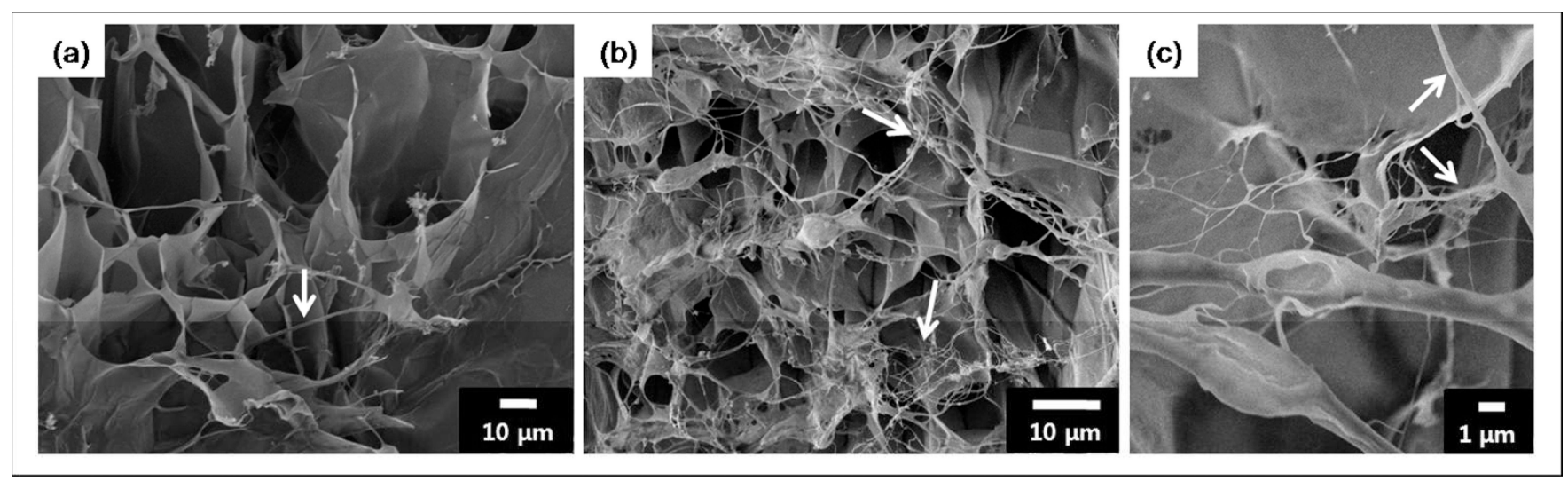

Figure 7.

Morphology of encapsulated hMSCs in (a) GelMA and (b) CNT-GelMA hydrogels with 0.5 $\mathrm{mg} / \mathrm{ml} \mathrm{CNTs}$. (a) Cytoplasmic prolongations (arrow) from the flat cell body of a hMSC extends to the over surface of the pores. (b) Thin threadlike cytoplasmic prolongations (arrows) that extend from the round bodies of hMSCs. (c) Magnified image showing the details of long threadlike cytoplasmic prolongations (arrows) that extend from the round body of a single hMSC, interweave with and reach nanofibers. 


\section{Table 1}

The compressive modulus and spreading behavior of encapsulated cells within CNT-GelMA and other hydrogels.

\begin{tabular}{ccccc}
\hline Sample & $\begin{array}{c}\text { Compressive modulus } \\
(\mathbf{k P a})\end{array}$ & Cell type & $\begin{array}{c}\text { Days at } \\
\text { spreading }\end{array}$ & Spreading \\
\hline $5 \%$ GelMA & $10 \pm 0.5$ & $3 \mathrm{~T} 3$ fibroblast & 2 & + \\
$0.5 \mathrm{mg} / \mathrm{ml}$ CNT- & $31 \pm 2.4$ & $3 \mathrm{~T} 3$ fibroblast & 2 & + \\
GelMA hydrogel & & & & + \\
$\begin{array}{c}10 \% \text { and } 15 \% \\
\text { GelMA hydrogel16 }\end{array}$ & $15-30$ & $3 \mathrm{~T} 3$ fibroblast & - & - \\
$\begin{array}{c}\text { GelMA/PEG } \\
\text { hydrogel17 }\end{array}$ & 80 & 3T3 fibroblast & 7 & + \\
HA-PEG37 & 48 & MSC & 21 & + \\
$\begin{array}{c}\text { Alginate } \\
\text { hydrogel38 }\end{array}$ & 1.5 & HepG2 & - & - \\
\hline
\end{tabular}

